Article

\title{
Research on Optical Properties of Tapered Optical Fibers with Liquid Crystal Cladding Doped with Gold Nanoparticles
}

\author{
Joanna E. Moś ${ }^{1, * \mathbb{C}}$, Joanna Korec ${ }^{1}$, Karol A. Stasiewicz ${ }^{1}$, Bartłomiej Jankiewicz ${ }^{2}{ }^{\mathbb{D}}$, \\ Bartosz Bartosewicz $^{2}$ and Leszek R. Jaroszewicz ${ }^{1}$ \\ 1 Institute of Technical Physics, Military University of Technology, 00-908 Warsaw, Poland; \\ joanna.korec@wat.edu.pl (J.K.); karol.stasiewicz@wat.edu.pl (K.A.S.); leszek.jaroszewicz@wat.edu.pl (L.R.J.) \\ 2 Institute of Optoelectronics Military University of Technology, 00-908 Warsaw, Poland; \\ bartlomiej.jankiewicz@wat.edu.pl (B.J.); bartosz.bartosewicz@wat.edu.pl (B.B.) \\ * Correspondence: joanna.mos@wat.edu.pl; Tel.: +48-261-837-001
}

Received: 15 May 2019; Accepted: 12 June 2019; Published: 14 June 2019

check for updates

\begin{abstract}
This paper presents results obtained for biconical tapered fibers surrounded/immersed in liquid crystal mixtures. The phenomenon of light propagating in the whole structure of a tapered fiber allows the creation of a sensor where the tapered region represents a core whereas the surrounding medium becomes a cladding. Created devices are very sensitive to changing refractive index value in a surrounding medium caused by modifying external environmental parameters like temperature, electric or magnetic field. For this reason, the properties of materials used as cladding should be easily modified. In this investigation, cells have been filled with two different nematic liquid crystals given as $1550^{*}$ and 6 CHBT (4-(trans-4-n-hexylcyclohexyl) isothiocyanatobenzoate), as well as with the same mixtures doped with $0.1 \mathrm{wt} \%$ gold nanoparticles (AuNPs). Optical spectrum analysis for the wavelength range of 550-1150 $\mathrm{nm}$ and time-courses performed for a wavelength of $846 \mathrm{~nm}$ at the temperature range of $\mathrm{T}=25-40{ }^{\circ} \mathrm{C}$ were provided. For all investigations, a steering voltage in the range of 0-200 V which allows establishing the dopes' influence on transmitted power and time response at different temperatures was applied.
\end{abstract}

Keywords: liquid crystal; gold nanoparticles; optical fiber device; tapered optical fiber

\section{Introduction}

Due to the rapid development of optical fiber technologies, we reach limits related to the use of materials or arrangements to measure different factors. We are looking for the possibility of using a "new material" to get an improvement in devices' parameters or to increase its sensitivity. For many years, scientists have been looking for and using combinations of different materials, using specific properties of each of them [1-3]. As a result, modified materials have been produced with new properties called hybrids. One of the interesting technological connections is the use of the fiber optic and liquid crystal technology [4,5]. This hybrid combination allows us to create an active, functional element consisting of a passive element-an optical fiber and an active liquid crystal (LC) medium, which properties can be controlled through electric field and temperature [6]. An important aspect is to find a common feature for these materials and their mode of interaction. In both cases, the common feature is the refractive index parameter of glass and LC, respectively. Use of optical fiber was related to the method of light transmission. Propagation of light in the fiber is associated with an appropriate selection of materials forming the cladding and the core with an appropriate refractive index $\mathrm{n}_{\text {core }}, \mathrm{n}_{\text {cladding }}$ filling the Maxwell equation/condition, as well as Snell law. The light beam can be 
propagated in two ways: based on phenomena of a total internal reflection (TIR) (in classic fibers) [7] or the principle of photonics' bandgap (in photonic crystal fibers, PCF) [8]. The change in the refractive index of materials caused modified properties of the propagated light in the form of modes. The value of the normalized frequency $\mathrm{V}$ determined the number of modes propagating in the fiber and the losses. This parameter is described as [9]:

$$
V^{2}=a \frac{2 \pi}{\lambda} \sqrt{n_{\text {core }^{2}-n_{\text {cladding }}^{2}}}
$$

From the above formula, it can be concluded that the number of modes propagating in the fiber is also influenced by the wavelength $\lambda$ and the diameter of the core a. This parameter is important when we want to use a tapered optical fiber [10-12] or long period gratings (LPG) $[13,14]$ as an element of optical devices. LCs are built of elongated molecules. Such construction allows determination of the anisotropic properties of LC, characterized by ordinary $n_{o}$ and extraordinary $n_{e}$ reflective indices. Depending on the light propagation direction, a different value of the refractive index will be detected. Through an appropriate electrical field (higher than threshold voltage) or temperature (changes in structure), we can change the reflective index by reorientation of LC position. While combining these materials, we notice that the optical fiber will be sensitive to changes in the LC refractive index [15]. Considering the diversity of optical fibers and LC types, there are many combinations possible. Many works have been written about fulfilling the air holes of a PCF with LC which causes a change of effective refractive index of the cladding structure and, hence the change of the propagation properties [16,17]. LCs can also be used externally by constructing a liquid crystal cell (LCC) with a tapered optical fiber or LPG placed between the cell's plates $[13,14]$. It is important to choose an appropriate LC with its refractive index, transition temperature to the isotropic state, dielectric permittivity, structure, composition, etc. Selection of these parameters affects the parameters of later devices, e.g., time of relaxation, threshold voltage, etc. As a result, we obtain interesting devices' sensors for single factors, as well as optical filters for a chosen wavelength $[13,18]$. The combination of these materials provides many benefits: optical fibers can be used over long distances, device miniaturization, device tunability in a different wave range and manufacturing low costs.

The use of a tapered optical fiber and LCs has been known for many years [19,20]. It was possible to control LCs, but they were characterized by high threshold voltages, as well as by large thicknesses of LCCs. Nowadays, the newly developed technology associated with the parameters' improvement is used as a novelty-use of appropriate spacers close to the taper waist diameter, use of the LC optimal type, or use of a LC mixture with other materials such as nanoparticles [1-5]. The research was carried out on the influence of doping with gold nanoparticles on LCC properties. As a result, improvements were made to such parameters as decreased threshold voltage and response time of the LCC [3]. Our research team has investigated how nanoparticles with LC affect light propagation in the optical fiber and, more specifically, in a tapered optical fiber, as well as how to elaborate a new kind of device working in a different wave range. In the research LCs named 1550 and 6CHBT (4-(trans-4-n-hexylcyclohexyl) isothiocyanatobenzoate) were applied-well known from filling air holes in PCF fibers [21]. Novelty of these works is connected with liquid crystal doping nanoparticles and applying them as a cladding with a possibility of refractive index steering. Previous literature on the use of a liquid crystal mixture with nanoparticles as a cladding of the optical fiber taper was not found. For this reason, the presented results are unique. Tapers were manufactured from a standard telecommunication single-mode fiber (SMF) with a cut-off wavelength of $1260 \mathrm{~nm}$-the most popular and low-cost material produced by Corning ${ }^{\circledR}$. Thanks to the tests carried out on cells with pure LC and with a mixture of gold nanoparticles with LC, we can compare the results of adding nanoparticles to light propagation under voltage and temperature control. 


\section{Materials and Methods}

\subsection{Propagation of Light in Tapered Optical Fiber with and without Liquid Crystal Cladding}

An optical fiber consists of two concentrically arranged dielectrics: the core and the cladding. The core is characterized by a higher refractive index than the cladding [8]. It determines light propagation in that structure based on a total internal reflection (Figure 1). The light is propagated in the form of modes in the core and a small part of energy penetrating the cladding which is named the evanescent field. During the technological process consisting of heating a certain part of the fiber and pulling it out, a tapered optical fiber can be created. The result of a fiber's tapered structure is change in the core and cladding diameters (Figure 2). As a result, the field of the basic mode occupies an increasingly larger cross-section of the cladding until it reaches the point where it is guided in its entire cross-section. As a result, the field of the basic mode occupies an increasingly larger cross-section of the cladding until it reaches the point where it is guided in its entire cross-section.

a)

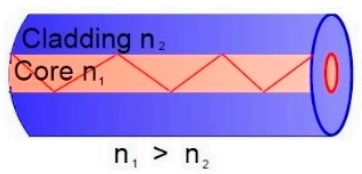

b)

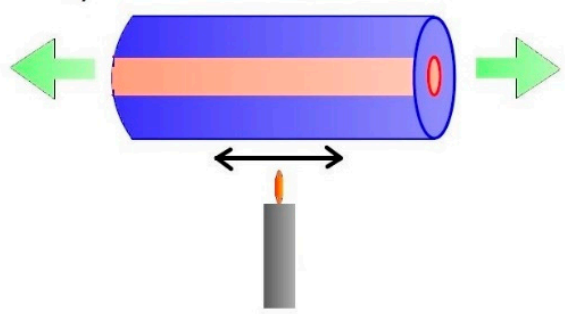

Figure 1. (a) Light propagation in optical fiber and (b) process of heating and elongation of the optical fiber.

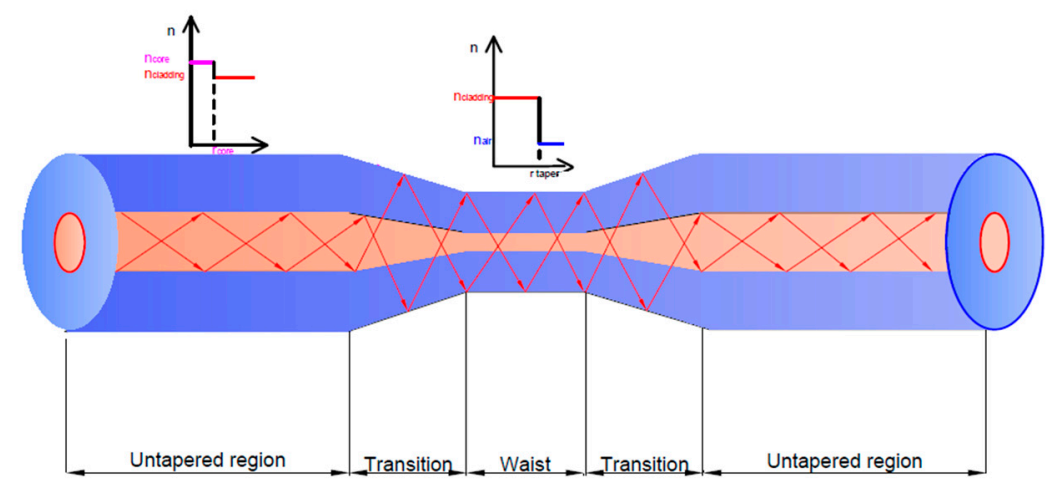

Figure 2. The scheme of light propagation in a tapered optical fiber-changes of boundary conditions.

The change of boundary conditions is also directly related to the change of optical fiber dimensions. The light is not propagated at the core/cladding interface, but the air/cladding interface. The approximations used in the mode solutions for a standard optical fiber, where the difference in refractive indices is small (lower than $1 \%$ ), are not applicable. In this case, in the tapered optical fiber, the light beam stays to be exposed to influence by the fiber surroundings — the light beam is propagating in a whole structure (as a core) and the surroundings become a cladding as is shown in Figure 2 [8,9].

Such an approach allows us to use additional material in a tapered area especially in the taper waist region (Figure 3). In this region, the optical fiber will be sensitive to external changes of the reflective index of the material that surrounds the fiber. Such connections with various materials have been widely used to build sensors, filters, and amplifiers [22,23]. 

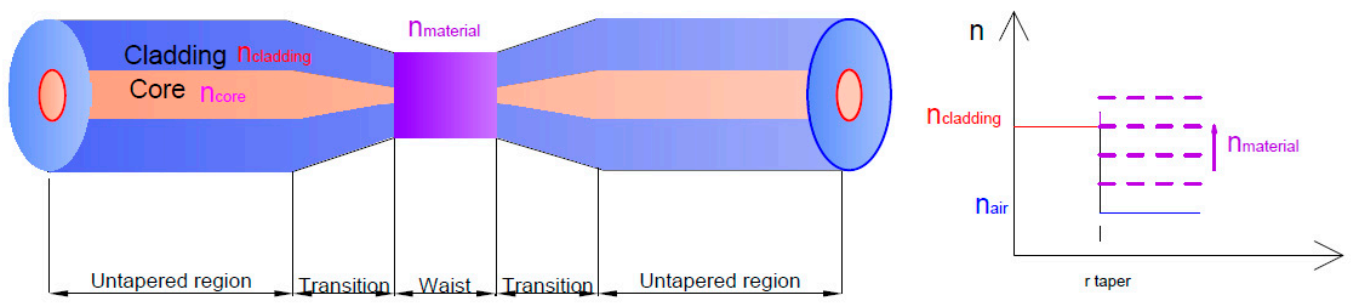

Figure 3. The tapered optical fiber with extra material.

The reason for taking liquid crystals as a research object is that they are anisotropic materials possessing variable refractive index depending on the molecules' orientation. It should also be mentioned that in many cases LCs have a higher refractive index than an optical fiber (propagation changes from total internal reflection to the band gap propagation or a mix of them for different wavelengths) which makes them most interesting materials. Additionally, LCs' substrates create a resonance device which causes the situation that some part of the light is reflected from the glass with alignment layers and can be coupled back to the fiber taper. In a standard single-mode fiber (SMF) the basic mode is propagated. When we taper such a fiber, boundary conditions change and we can see many modes propagating especially in the taper waist area. An attitude mode begins to couple with cladding modes so that the light beam is modified. The light starts to leak from the taper's structure to the liquid crystal cladding as shown in Figure 4. In LC, light beams are limited by the glass with alignment layers as it was mentioned earlier. Depending on the used voltage, the angle of LC scattering increases [see Figure $4 b][15,24]$.

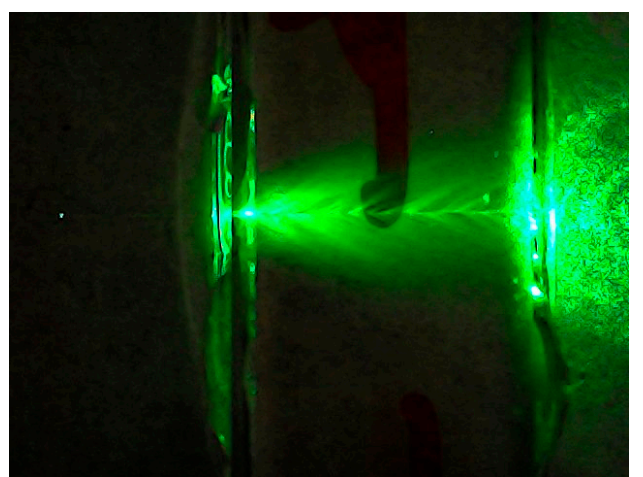

(a)

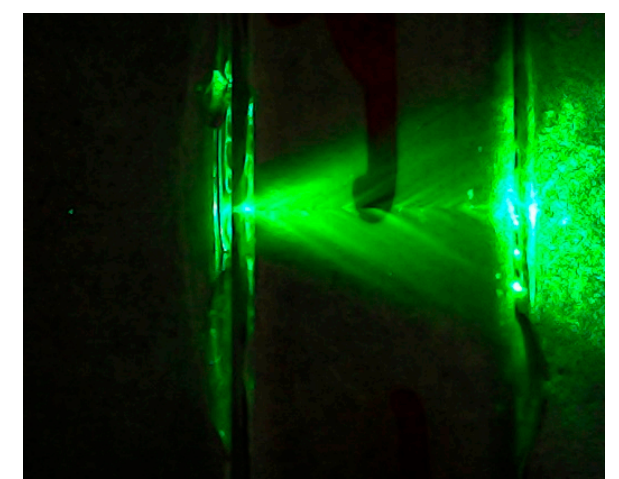

(b)

Figure 4. Light propagation in the liquid crystal cell with the tapered fiber: (a) $U=0 \mathrm{~V}$; (b) $U=200 \mathrm{~V}$.

\subsection{Materials}

In this paper were investigated two nematic LCs: 6CHBT (4-(trans-4-n-hexylcyclohexyl) isothiocyanatobenzoate) and $1550^{*}$, also the same mixtures doped with $0.1 \mathrm{wt} \%$ AuNPs. Table 1 presents the main properties of the pure LCs used in this paper. 6CHBT LC is a low melting LC and compared to the $1550^{*}, 6 \mathrm{CHBT}$ LC contains only one mixture component. Also, there is a significant difference between the refractive indices of both LCs. As presented in Table 1, 6CHBT has a much higher refractive index than $1550^{*}$ and is higher than silica glass in room temperature. According to the literature, in some cases, the light can be propagated in the core with a lower refractive index than the effective cladding refractive index $[23,25]$. 
Table 1. Properties of the used liquid crystals (LCs): 6CHBT (4-(trans-4-n-hexylcyclohexyl) isothiocyanat obenzoate) and $1550^{*}[21,26]$.

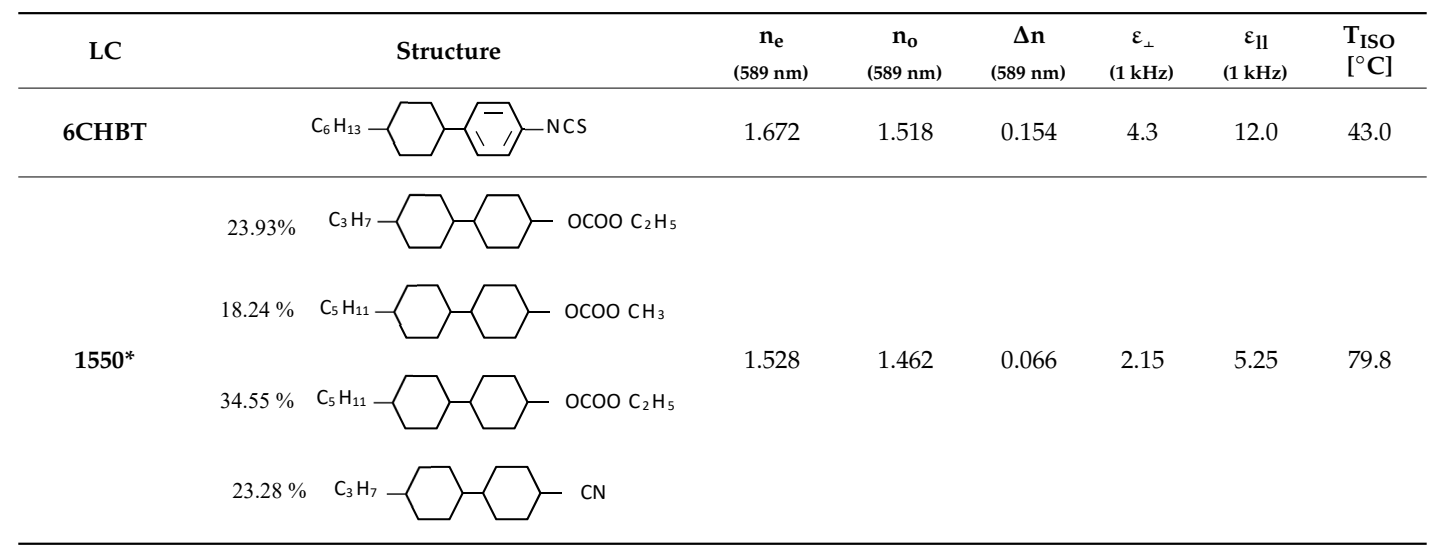

Basing on the results from [27], the authors decided to mix pure LCs with 0.1 wt $\%$ AuNPs. Preparation of nanoparticle (NP)-doped LCs has been carried out in the same manner as described in detail elsewhere [28]. Briefly, the colloidal suspension of Au NPs in an organic solvent was prepared using the Brust-Schiffrin method [29]. However, instead of toluene, we have used chloroform. After synthesis and purification, we have obtained suspension of Au NPs in chloroform with a concentration of $1.2 \mathrm{mg} / \mathrm{mL}$. Au NPs had diameters in the range of $1-3 \mathrm{~nm}$. To obtain NPs-doped LCs with various $\mathrm{Au}$ NPs content, we have mixed various volumes of the colloidal suspension of AuNPs with liquid crystal and evaporated chloroform at $50{ }^{\circ} \mathrm{C}$ for $48 \mathrm{~h}$.

\subsection{Technology}

This section presents the manufacturing of an LCC with the tapered optical fiber. The FOTET (fiber optic taper element technology) system (see Figure 5) was used for tapered fibers' manufacturing. FOTET is a dedicated device for preparing different optical fiber elements, e.g., couples, tapers or isolators. The main advantage of this technique is the possibility to modify different types of optical fibers, e.g., single mode, multimode, as well as photonic crystal fibers [30] and to obtain different shapes of tapers: adiabatic, as well as non-adiabatic.

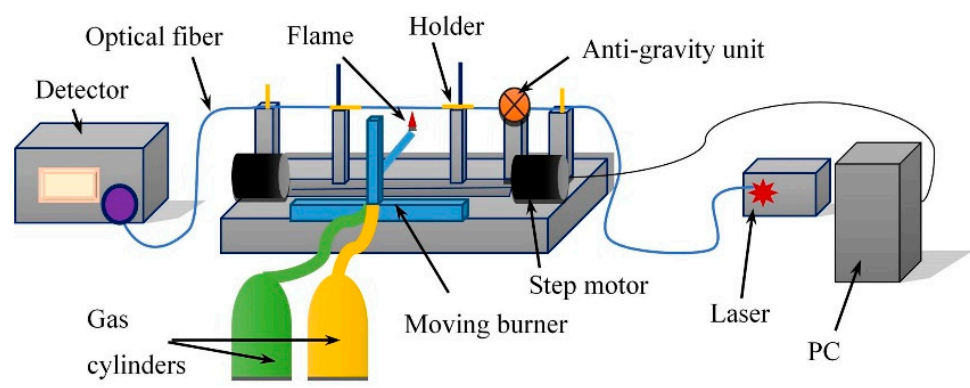

Figure 5. Scheme of the FOTET (fiber optic taper element technology) system's arrangement of the tapered fiber manufacturing.

Tapering is performed by stretching the optical fiber above the flame from a low-pressure burner powered with a propane-butane-oxygen gas mixture. An appropriate proportion of this mixture and distance between flame and fiber allow for controlling flame temperature and, connected with it, drawing velocity. In this process, the temperature is most important because too high a temperature causes melting of the optical fiber and too low a temperature is not enough to soften the fiber, which results in stopping the stretching process. Appropriate temperature is the softening temperature of glass (around $1200^{\circ} \mathrm{C}$ ). A pure mixture of propane-butane and oxygen gases allows to obtain a 
homogenous and adequate temperature distribution. The velocity of fiber elongation is controlled by software which is based on a measurement of the transmitted optical signal magnitude. Additionally, the special anti-gravity unit is used because during the elongation process the fiber position is reduced. The signal increase in the anti-gravity system is an impulse for changing drawing velocity, as well as lowering the burner position [11,12]. Additionally, by selection of a proper flame, we can make a taper with a very small dimension of the taper waist. The main advantage of preparing tapered fibers on the FOTET are full control elongation parameters like elongation length, burner movement, and its distance from the fiber or step motors velocity, taper waist region diameter. Additionally, during elongation, changing optical power is constantly monitored by using a laser and a photodetector connected to the opposite ends of the fiber. Thus, attenuation after the finished tapering process can be easily calculated.

Prepared tapers used for manufacturing devices are characterized by low losses below $\alpha=0.2 \mathrm{~dB}$, elongation $\mathrm{L}=20.20 \pm 0.05 \mathrm{~mm}$, and diameter $\varphi=14.50 \pm 0.50 \mu \mathrm{m}$ for a wavelength of $1550 \mathrm{~nm}$ which corresponds to the single mode work of the used single mode fiber.

In the next stage, the tapered fiber LCCs were prepared. The initial arrangement of molecules is achieved by using a photoalignment poly(estermide) layer on the glass substrate covered with ITO (Indium tin oxide) (further called electrode) [31]. The obtained tapered fiber is placed on the electrode as close as possible but without touching it. The position is obtained with control of a CCD (Charge Coupled Device) camera with special objective lenses. Any contact between the tapered fiber and the electrode causes radiating out optical power and increasing losses. To maintain the same distance between electrodes, spacers with a diameter of $\varphi=40 \mu \mathrm{m}$ were used. In this paper, the perpendicular arrangements of taper and electrodes were investigated. In this case, the top and bottom electrodes have a perpendicular direction of the alignment layer with respect to the fiber/taper axis. Prepared cells are filled with LCs right before the measurement to ensure conglomeration on the NP. Figure 6 presents the scheme and cross-section of the prepared cell.

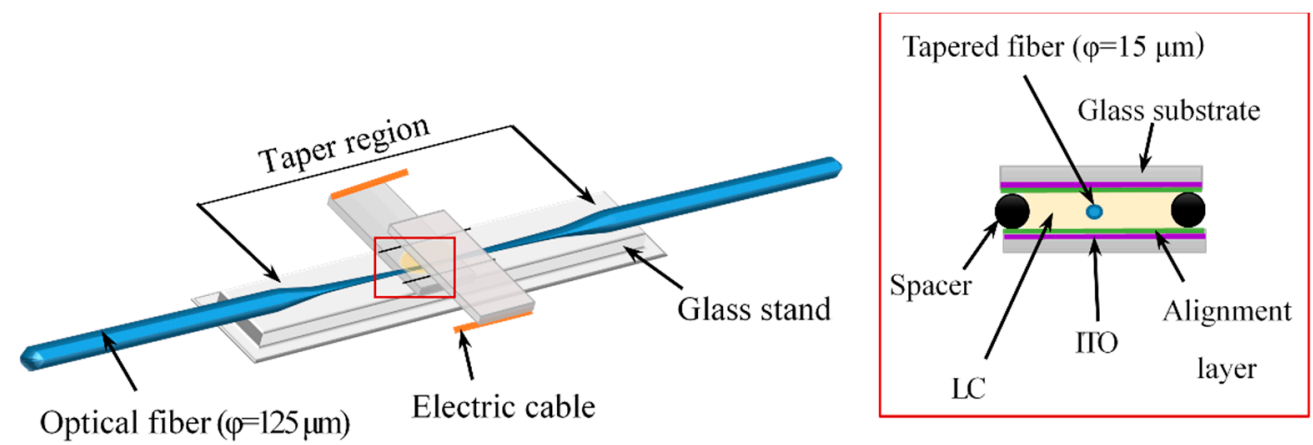

Figure 6. Scheme of the liquid crystal cell with a tapered fiber.

In general, the different refractive index of a waveguide cladding (LC around tapered fiber) depends on the orientation of n-director molecules in the external electric field. However, around the taper, an area of disoriented LC molecules is formed which is attached to the fiber as it is shown in Figure 7 [32]. This part of the LC cannot be reoriented even with a high voltage, which is connected to adhesive strength between glass and LC molecules. Nevertheless, its diameter is smaller than the wavelength. Hence, it will not be under investigation in this paper. It also should be noticed that in this investigation only the medium refractive index, which can be described as the medium molecules' director, is taken under consideration. In the doped LC topological defects are induced around nanoparticles (See Figure 7). Even small amounts of NPs significantly influence the properties of bulk material due to intermixing NP effects on alignment layers and bulk properties [33]. Additionally, we can predict that in a temperature change process NPs will be new small centers of expanding temperature. 


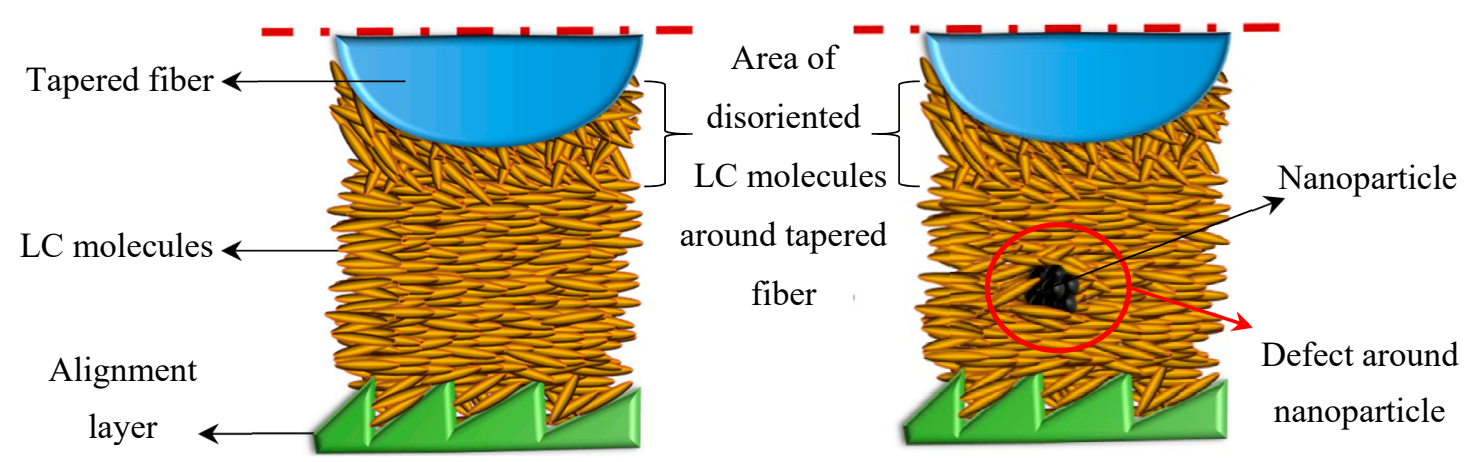

(a)

(b)

Figure 7. Scheme of molecules' orientation around the taper and in the whole volume of the LC cell: (a) pure LC; (b) LC doped with nanoparticles.

Results obtained show the positive and negative influence of dopants. Nanoparticles' behaviour in these liquid crystals can be explained by the difference between physical and chemical properties of 6 CHBT and 1550*. In contrast to 6CHBT, $1550^{*}$ is a mixture of four components with much higher molar mass than the first one. The differences in measured properties are mainly connected to the concentration and size of nano-molecules in the solution.

\section{Results}

Applied voltage influence on changes in spectral characteristics was investigated. Figure 8 shows the scheme of an optical system for measuring changes in spectral characteristics depending on voltage. For the measurement a high-power supercontinuum laser (SuperK EXTREME, NKT Photonics, Southampton, UK) (since light operates in the wavelength range of 400-2400 nm), an optical spectrum analyser with the spectral range of 350-1200 nm, (AQ6373, Yokogawa, Tokyo, Japan), signal generator (DG1022, RIGOL Beijing; China), and voltage amplifier (A400D, FLC Electronics, Molndal, Sweden) were used.

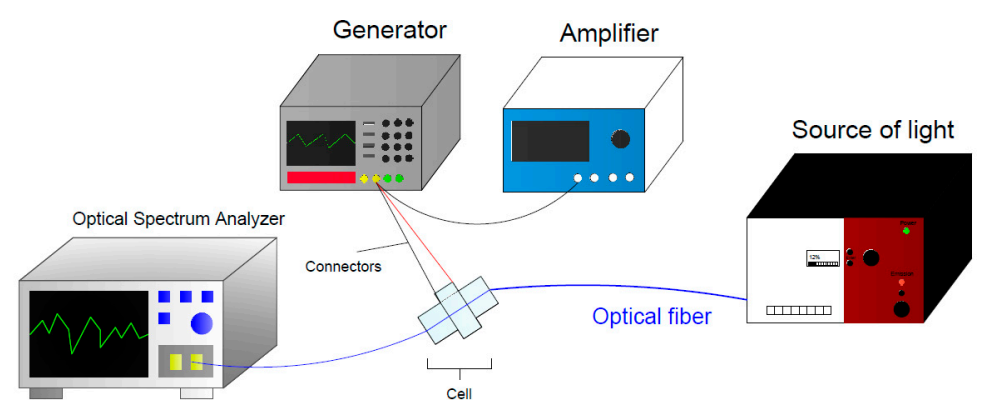

Figure 8. Scheme of the optical system for measuring changes in spectral characteristics depending on voltage.

Figure 9 presents the spectral characteristics for a cell filled with $1550^{*}$ and 6CHBT LC for pure and doped NPs with voltage steering without modulation in the range of $0-200 \mathrm{~V}$ at room temperature. For $1550^{*} \mathrm{LC}$, a reduction of power transmission in almost whole wave range can be observed. Steering by an electric field causes an increase of power light propagated in the optical fiber device as can be seen in Figure 9a,b. Much better transmission is observed for 1550* doped Au NPs. In all wave ranges, we observe smaller losses than for pure LC. Together with applying voltage power in the full range, optical power is increasing and, additionally, broadening the wave range can be observed in comparison to the pure LC. For pure 6CHBT we observe smaller attenuation of optical power in whole range than for pure $1550^{*}$ mixture. Furthermore, changes in optical power regarding the applied electric field are 
insignificant. For 6CHBT with nanoparticles, interference between modes are observed in Figure 9d (magnification of the chosen part of spectral characteristics) which is not observed for $1550 *$ mixture.

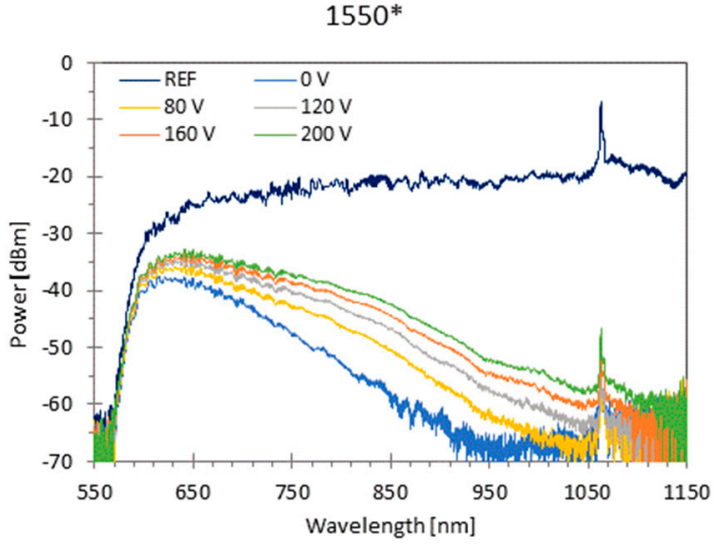

(a)

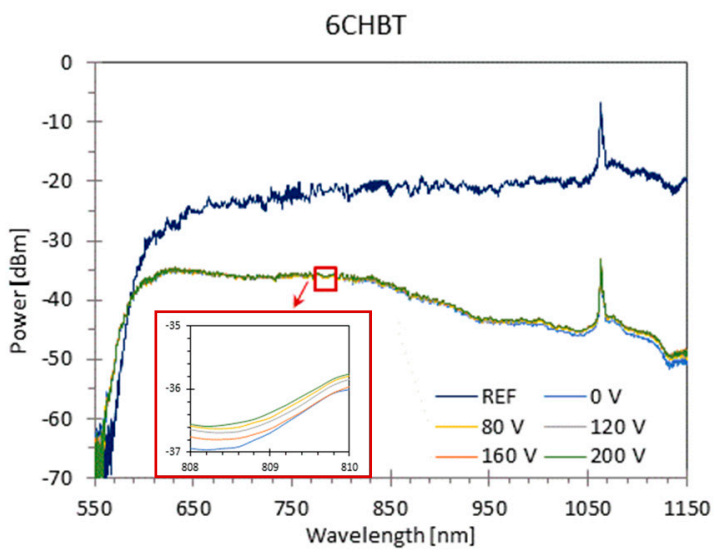

(c)

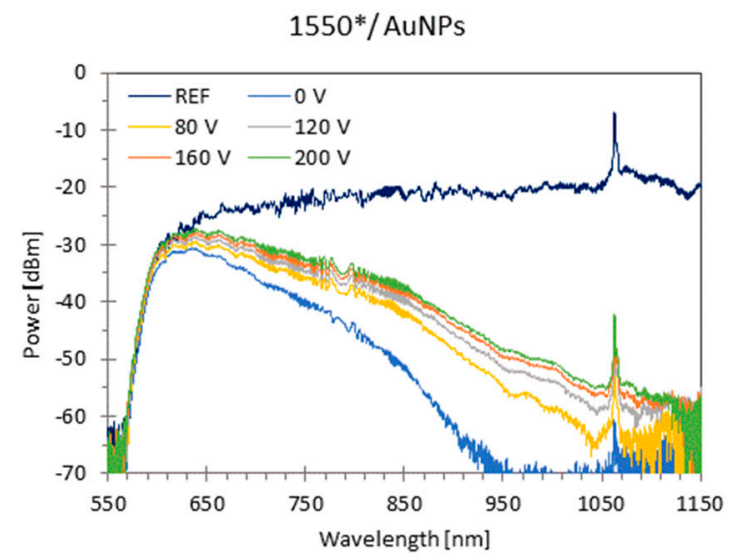

(b)

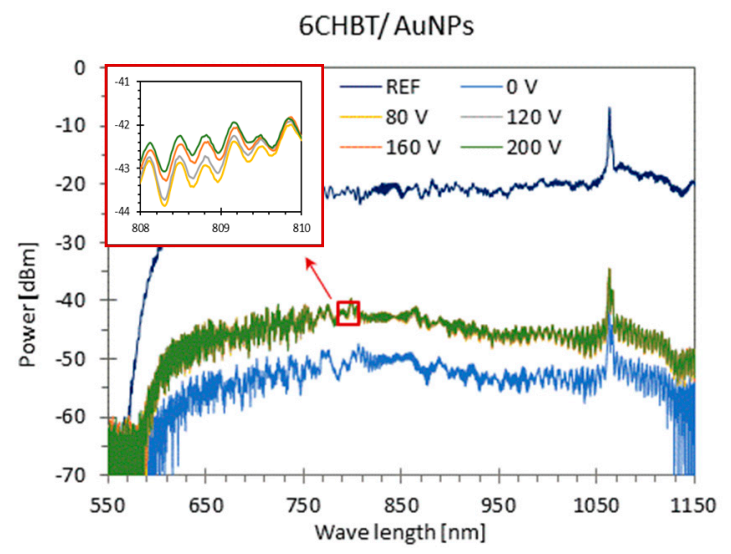

(d)

Figure 9. Spectra obtained for LCs: (a) 1550* (pure); (b) 1550* + $0.1 \mathrm{wt} \%$ AuNPs; (c) 6CHBT (pure); (d) $6 \mathrm{CHBT}+0.1 \mathrm{wt} \%$ AuNPs, for different steering voltage in the range U of 0-200 V.

If we apply additional modulations of electrical signal—the signal's shape and frequency-we notice that this is mapped on the spectral characteristics. We can select and amplify certain ranges of wavelengths. In practice, this can be used as a bandpass filter. When comparing results for pure LC and with nanoparticles for $1550^{*}$, the result is more visible and can be observed for a wider range over $50 \mathrm{~nm}$. Results for a 6CHBT donation of NPs destroy the possibilities of a transfer modulated signal (see Figure 10).

The application of different LC mixtures influences speed of device operation and answer time to measurement parameters). The scheme of the optical system for measuring time courses depending on voltage and temperature is shown in Figure 11. For the measurement we used as a source of light a single mode fiber pigtailed laser (LP852-SF30, ThorLabs, Newton; USA) operating at the center wavelength of $846 \mathrm{~nm}$, a signal generator (DG1022, RIGOL, Beijing; China), voltage amplifier (A400D, FLC Electronics, Molndal; Sweden), an Si-amplified detector operating in the wavelength range of 350-1100 nm, and a climatic chamber (VCL 7010, Votsch Industrietechnik, Balingen, Germany) for temperature measurement in the temperature range of -70 to $180{ }^{\circ} \mathrm{C}$. 


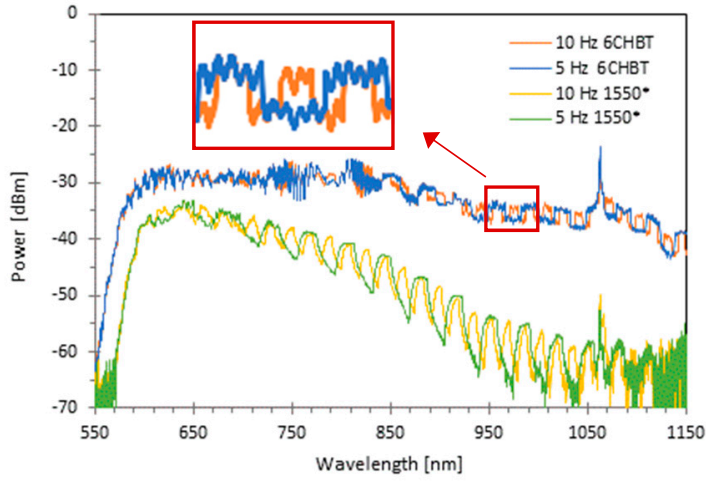

(a)

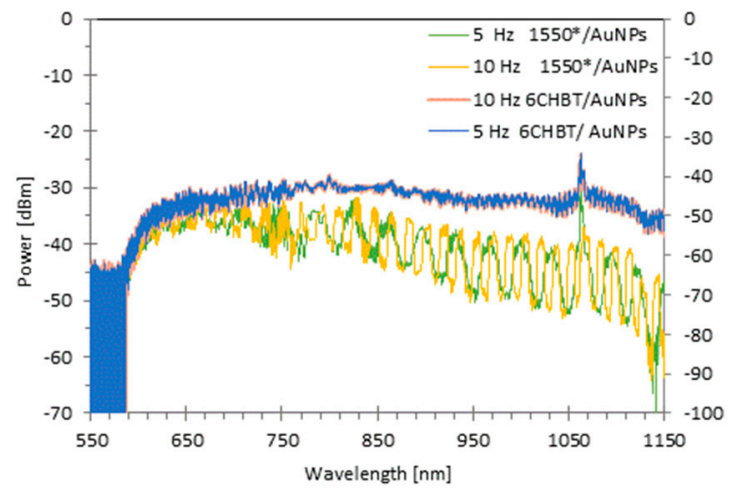

(b)

Figure 10. Spectral characteristics in the wavelength range of 550-1150 nm for applied $200 \mathrm{~V}$ signal amplitude. The square shape of modulation with a frequency of $5 \mathrm{~Hz}$ and $10 \mathrm{~Hz}$ has been applied (a) pure liquid crystals; (b) liquid crystals doped with $0.1 \mathrm{wt} \%$ AuNPs.

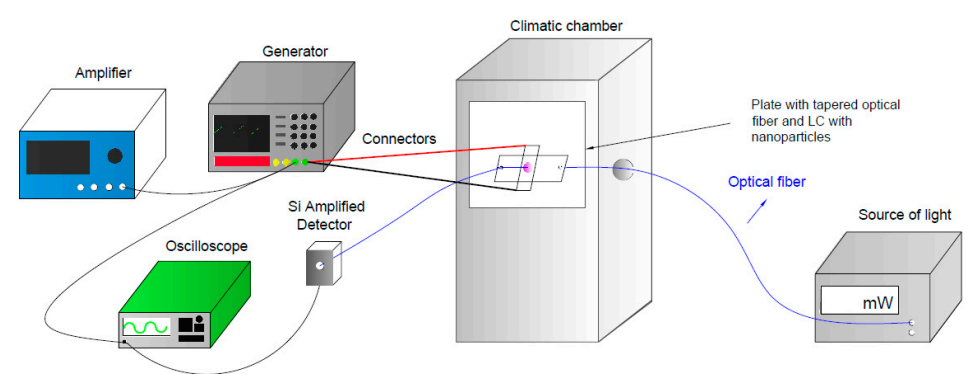

Figure 11. Scheme of the optical system for measuring time courses depending on voltage and temperature.

In a taper device with LC, answer time can be translated into the switching on and off times of the liquid crystal material (times of molecules director reorientation). Time courses obtained for a steering voltage range of $\mathrm{U}=80-200 \mathrm{~V}$ at room temperature $\left(25^{\circ} \mathrm{C}\right)$ are shown in Figure 12 . As can be seen, doping NPs to $1550^{*}$ mixture made a better image of square modulation with a higher power level. For all voltage measurement, switching times on and off can be easy to measure as shown in Figure 12a,b. For 6CHBT, doping NPs made increasing losses, the signal stays flat for pure and doped LC.

Investigation on LC devices was also provided for temperature dependence (see Figure 13). For both LC $1550^{*}$ and $6 \mathrm{CHBT}$ we measured temperature in the range of $25-40{ }^{\circ} \mathrm{C}$ with a square modulation of $10 \mathrm{~Hz}$ and a voltage steering of $200 \mathrm{~V}$ which determined full molecules' reorientationabove this voltage, there was no increase of power level. 
$1550^{*}$

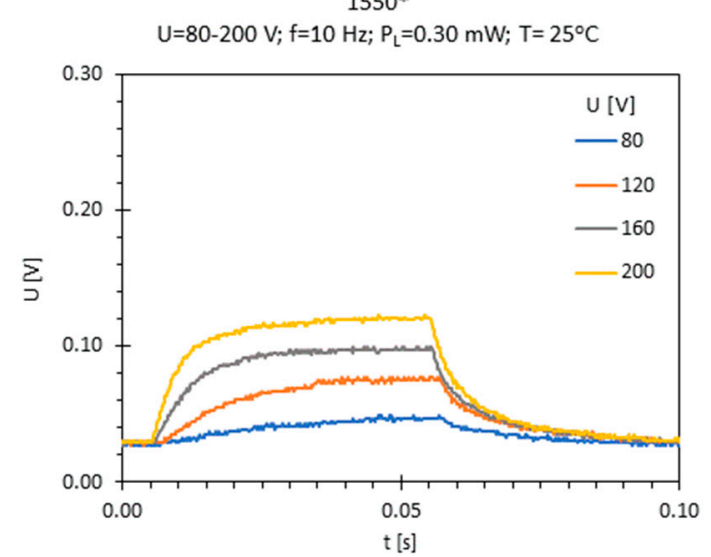

(a)

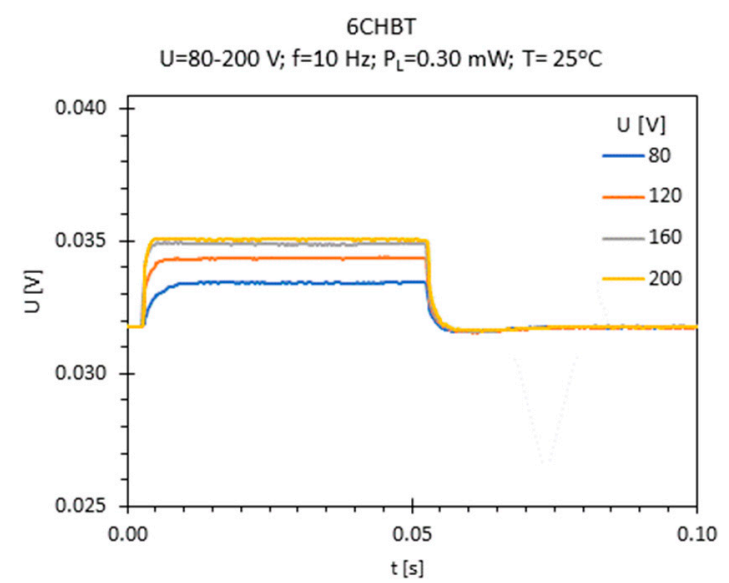

(c)
$1550 * /$ AuNPs

$\mathrm{U}=80-200 \mathrm{~V} ; \mathrm{f}=10 \mathrm{~Hz} ; \mathrm{P}_{\mathrm{L}}=0.30 \mathrm{~mW} ; \mathrm{T}=25^{\circ} \mathrm{C}$

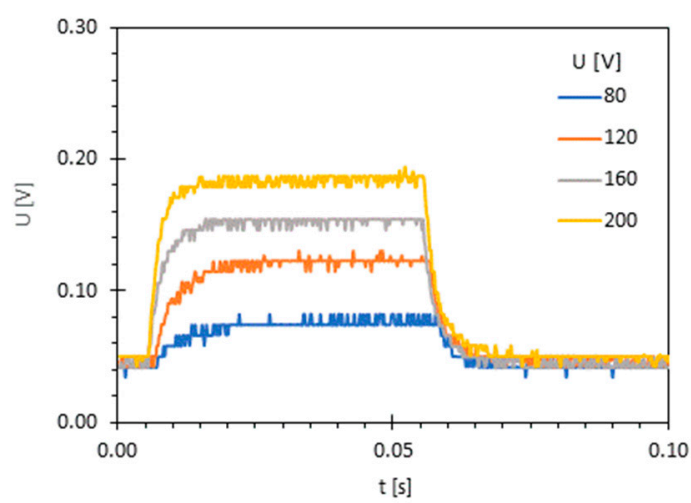

(b)

$6 \mathrm{CHBT} / \mathrm{Au}$ NPS $U=80-200 \mathrm{~V} ; \mathrm{f}=10 \mathrm{~Hz} ; \mathrm{P}_{\mathrm{L}}=0.30 \mathrm{~mW} ; \mathrm{T}=25^{\circ} \mathrm{C}$

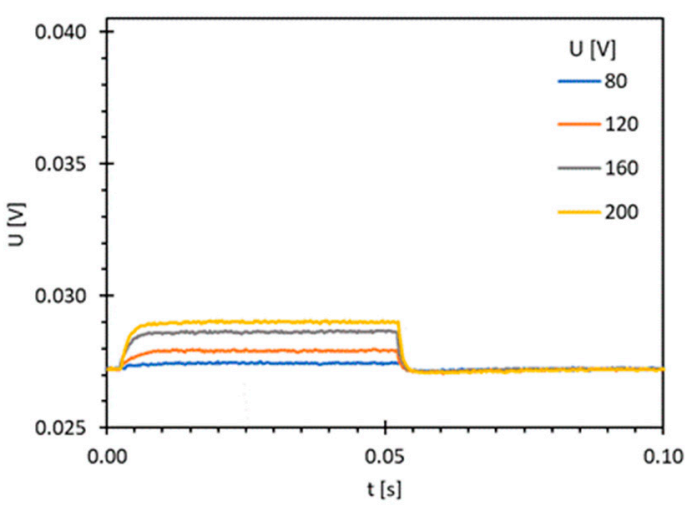

(d)

Figure 12. Time courses obtained for the steering voltage range of $\mathrm{U}=80-200 \mathrm{~V}$ at room temperature $\left(25^{\circ} \mathrm{C}\right.$ ) for: (a) $1550^{*}$ (pure); (b) 1550* $+0.1 \mathrm{wt} \%$ AuNPs; (c) 6CHBT (pure); (d) 6CHBT $+0.1 \mathrm{wt} \%$ AuNPs.

1550*

$\mathrm{U}=200 \mathrm{~V} ; \mathrm{f}=10 \mathrm{~Hz} ; \mathrm{P}_{\mathrm{L}}=0.30 \mathrm{~mW} ; \mathrm{T}=25-40^{\circ} \mathrm{C}$

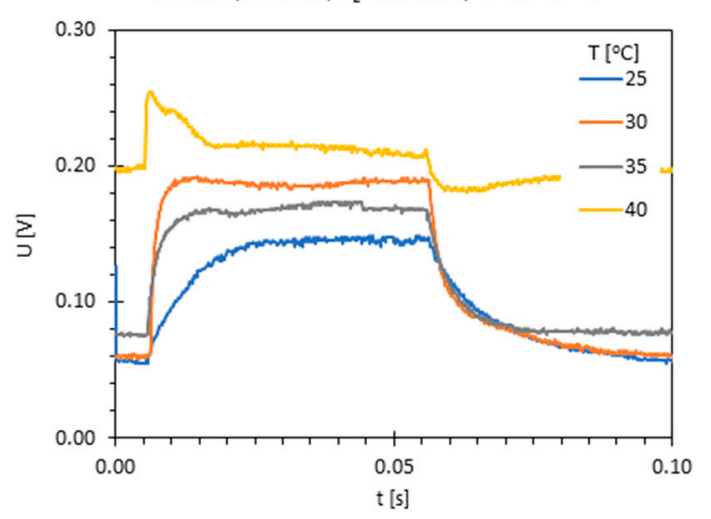

(a)
1550*/AuNPs

$U=200 \mathrm{~V} ; f=10 \mathrm{~Hz} ; P_{L}=0.30 \mathrm{~mW} ; T=25-40^{\circ} \mathrm{C}$

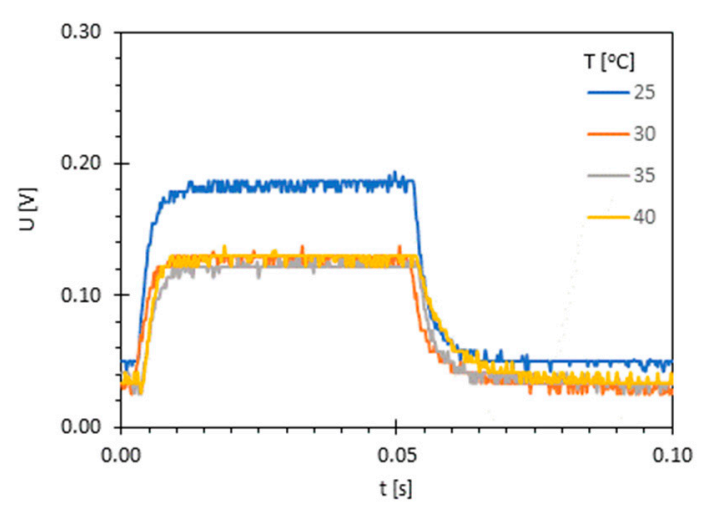

(b)

Figure 13. Cont. 


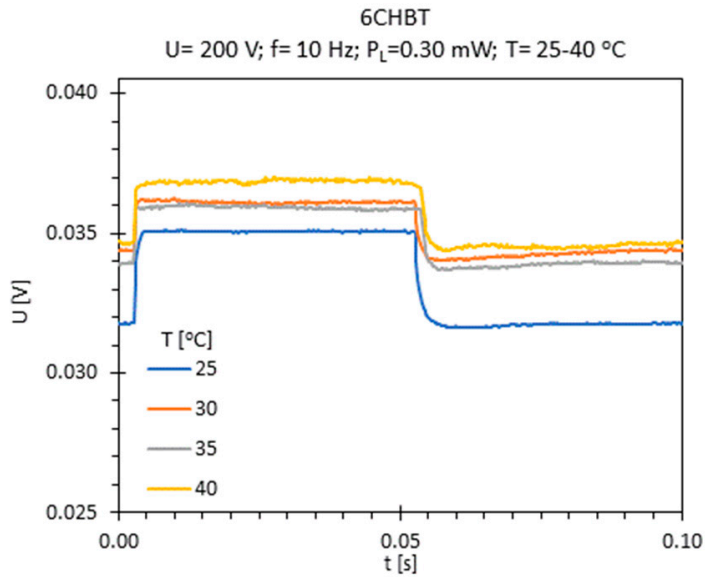

(c)

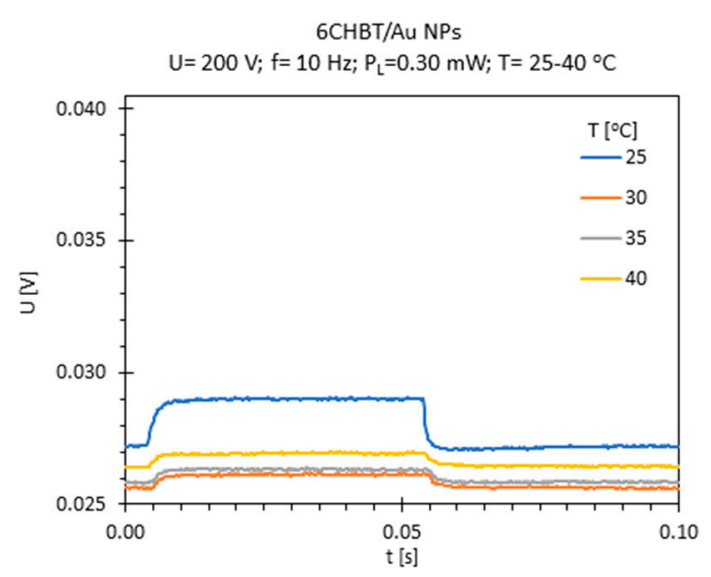

(d)

Figure 13. Time courses obtained for the steering voltage $\mathrm{U}=200 \mathrm{~V}$ at the temperature range of $\mathrm{T}$ = 25-40 ${ }^{\circ} \mathrm{C}$ for: (a) $1550^{*}$ (pure); (b) $1550^{*}+0.1 \mathrm{wt} \%$ AuNPs; (c) 6CHBT (pure); (d) $6 \mathrm{CHBT}+0.1$ wt $\%$ AuNPs.

As can be seen in Figure 13, for 1550* LC together with a temperature power increase of transmitted light also increases. For higher temperature modulation, the signal mapped by the LC taper device does not follow the forcing signal. Such changes can be a result of the reduction of elastic and viscosity parameters between the molecules of the LC together with temperature increases, as well as a change of the effective value of the refractive index (medium molecules director). For the $1550^{*} \mathrm{LC}$ with NPs donation, we observed a step decrease of power for $30^{\circ} \mathrm{C}$, and above this temperature, there is no change of power level which can be strictly connected with the NPs and their influence on LC structure and molecules' direction. For pure and doped 6CHBT LC, we observed the inversion of a power level change. For pure LC together with increasing temperature, power increases, and for the doped one with temperature increase power decreases.

As a switch on (off) time we assume the time required for a change in the light transmission through the investigated liquid crystal cell from 10\% to $90 \%$ (or vice versa) of its maximal value when steering voltage is switched on (or off) (See Figure 14a,b).

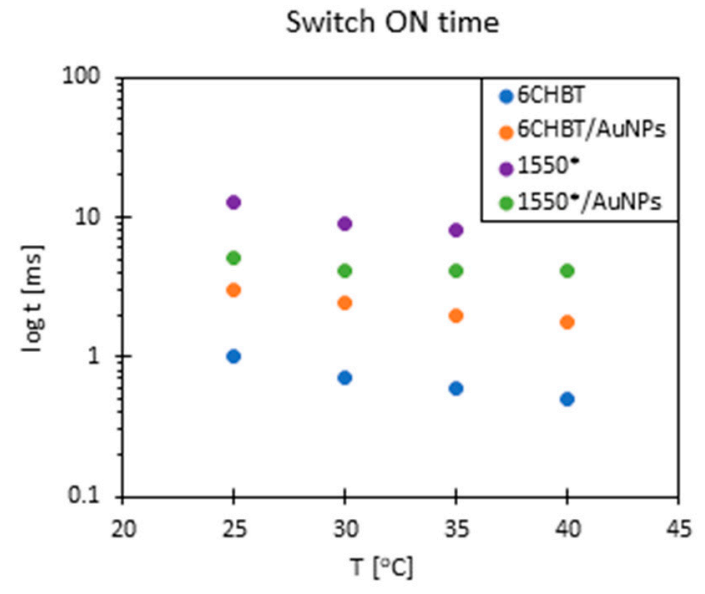

(a)

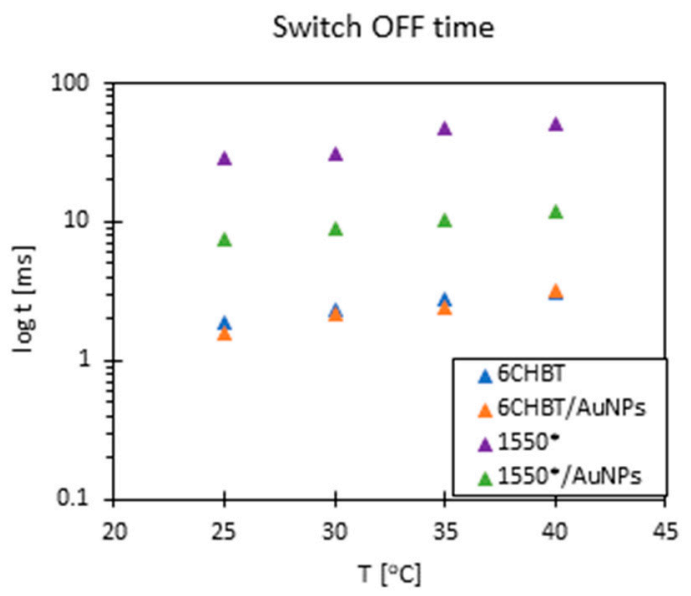

(b)

Figure 14. (a) Switch ON and OFF time for a for $1550^{*}$ and (b) $6 \mathrm{CHBT}$ (pure), $1550^{*}+0.1 \mathrm{wt} \%$ AuNPs; and $6 \mathrm{CHBT}+0.1 \mathrm{wt} \%$ AuNPs. 
For both cases, the applied pure $1550^{*}$ mixture possesses the highest switch on (Figure 14a) and switch off times (Figure 14b). By doping this mixture with Au nanoparticles, we obtain time decrease several times. For 6CHBT we can observe that switch on time for the doped LC increases over three times in contrast to the pure LC. Switching off times are almost on the same level in this liquid crystal.

Comparing the times of switch on and off of LC $1550^{*}$ and 6CHBT with nanoparticles, it can be observed that 6CHBT is much faster than the $1550^{*} \mathrm{LC}$ mixture which is connected with the viscosity strength of the chosen LC.

\section{Conclusions}

In this article, we present the possibility of manufacturing optical devices using two technologies: taper optical fibers and liquid crystals with gold nanoparticles. The optical fiber taper process allows for direct influence on propagating light beam inside the structure by changing the boundary condition of cladding parameters. This technology connected with LC materials, enables us to manufacture in-line miniaturized devices sensitive for many factors like temperature, electric, and magnetic field. Liquid crystals as materials possessing anisotropic properties are sensitive to changes of external factors: temperature, electric and magnetic fields. Any change in the external factor is associated with a change of the liquid crystal material properties. The light in the optical fiber taper enables a detection of the mentioned changes due to their sensitivity to a changing refractive index of the surrounding material. When we use only the electric field, we operate on the coefficient change in the $n_{e}-n_{o}$ range at constant temperature. When the temperature is changed, the values ne and no are simultaneously reduced. After exceeding the transition temperature to the isotropic state, $\mathrm{n}_{\text {iso }}$ is below the $\mathrm{n}$ of glass. Taking into consideration the conducted state of the art tests, we notice that as temperature rises, the spectral characteristics change as a result of structural change and such parameters of the liquid crystal as viscosity, refractive index, elastic. It is difficult to separate the result of the measured parameters in optical fiber measurements; however, in our case, it can be possible to separate two factors since we know the answer to a given temperature and voltage in approximation. Depending on the possible application, the working parameters for signal detection with different times of on/off switching can be improved. Connection of liquid crystals with nanoparticles allows for a significant change of their optical parameters, including effective refractive index (the medium molecule's director) and the materials' parameters like viscosity. LC is one of the materials which can be used as a functional material enabled to detect many factors like the electric or magnetic field or temperature. The results with LC and nanoparticles described show the possibility of manufacturing in line miniaturized devices for commercial application. By applying different kinds of LC mixtures, we can adjust the parameters of the devices to optimize their work depending of measurement requirements.

Author Contributions: Conceptualization L.R.J. and K.A.S.; methodology K.A.S.; J.E.M.; J.K.; B.J. and B.B., formal analysis J.E.M. and K.A.S., investigation J.E.M.; J.K. and K.A.S., data curation K.A.S. and L.R.J, writing-original draft preparation J.E.M.; J.K. and K.A.S., writing-review and editing L.R.J., visualization J.E.M. and J.K., supervision L.R.J. and K.A.S., funding acquisition J.E.M. and L.R.J.

Funding: This research was funded by National Science Centre, grant number UMO-2018/29/N/ST7/02347, Military University of Technology grant number RMN 08/689 as well as Statutory Task PBS 898 and supported by the Program of the Republic of Poland-Research Grant MUT project no. GBMON/13-995/2018/WAT in 2019.

Conflicts of Interest: The authors declare no conflict of interest.

\section{References}

1. Nealon, G.L.; Greget, R.; Dominguez, C.; Nagy, Z.T.; Guillon, D.; Gallaniand, J.L.; Donnio, B. Liquid-crystalline nanoparticles: Hybrid designand mesophase structures. Beilstein J. Org. Chem. 2012, 8, 349-370. [CrossRef] [PubMed]

2. Škarabot, M.; Ryzhkova, A.V.; Muševič, I. Interactions of single nanoparticles in nematic liquid crystal. J. Mol. Liq. 2018, 267, 384-389. [CrossRef] 
3. Hsu, C.J.; Lin, L.J.; Huang, M.K.; Huang, C.Y. Electro-optical Effect of Gold Nanoparticle Dispersed in Nematic Liquid Crystals. Crystals 2017, 7, 287. [CrossRef]

4. Woliński, T.R.; Siarkowska, A.; Budaszewskia, D.; Chychłowskia, M.; Czapla, A.; Ertman, S.; Lesiak, P.; Rutkowska, K.; Orzechowski, K.; Sala-Tefelska, M.; et al. Recent advances in liquid-crystal fiber-optics and photonics. Proc. SPIE 2017, 10125, 101250W. [CrossRef]

5. Kowerdziej, R.; Garbat, K.; Walczakowski, M. Nematic liquid crystal mixtures dedicated to thermally tunable terahertz devices. Liq. Cryst. 2018, 45, 1040-1046. [CrossRef]

6. Sengupta, A. Liquid Crystal Theory. In Topological Microfluidics: Nematic Liquid Crystals and Nematic Colloids in Microfluidic Enviroment; Springer: Basel, Switzeland, 2013; pp. 7-34.

7. Katsunari, O. Wave Theory of Optical Waveguides. In Fundamentals of Optical Waveguides; Academic Press: Londyn, UK, 2006; pp. 1-12.

8. Frazão, O.; Santos, J.L.; Araújo, F.M.; Ferreira, L.A. Optical sensing with photonic crystal fibers. Laser Photonics Rev. 2008, 2, 449-459. [CrossRef]

9. Brambilla, G. Optical fibre nanowires and mikrowires: Review. J. Opt. 2010, 12, 043001. [CrossRef]

10. Tian, Y.; Wang, W.; Wu, N.; Zou, X.; Wang, X. Tapered Optical Fiber Sensor for Label-Free Detection of Biomolecules. Sensors 2011, 11, 3780-3790. [CrossRef]

11. Moś, J.E.; Stasiewicz, K.A.; Garbat, K.; Morawiak, P.; Piecek, W.; Jaroszewicz, L.R. Tapered fibre liquid crystal tunable broad band filter. Phys. Scr. 2018, 93, 125002. [CrossRef]

12. Korec, J.; Stasiewicz, K.A.; Strzeżysz, O.; Kula, P.; Jaroszewicz, L.R. Electro-Steering Tapered Fiber-Optic Device with Liquid Crystal Cladding. J. Sens. 2019, 2019, 1617685. [CrossRef]

13. Chao, D.; Wang, Q.; Zhao, Y. Electrically tunable long period gratings temperature sensor based on liquid crystal infiltrated photonic crystal fibers. Sens. Actuators A Phys. 2018, 278, 78-84.

14. Czapla, A.; Bock, W.J.; Woliński, T.R.; Mikulic, P.; Dabrowski, R.; Nowinowski-Kruszelnicki, E. Electically tunable long-period fiber gratings with low- birefringence liquid crystal near the turn-around point. Opto-Electron. Rev. 2017, 25, 290-295. [CrossRef]

15. Choudhury, P.K.; Soon, W.K. On the tapered optical fibers with radially anisotropic liquid crystal clad. Prog. Electromagn. Res. 2011, 115, 461-475. [CrossRef]

16. Wahle, M.; Kitzerow, H.S. Liquid crystal assisted optical fibers. Opt. Express 2014, 22, 262-273. [CrossRef] [PubMed]

17. Woliński, T.R.; Szaniawska, K.; Ertman, S.; Lesiak, P.; Domański, A.W.; Dąbrowski, R.; Nowinowski-Kruszelnicki, E.; Wojcik, J. Influence of temperature and electrical fields on propagation properties of photonic liquid-crystal fibers. Meas. Sci. Technol. 2006, 17, 985-991. [CrossRef]

18. Larsen, T.T.; Bjarklev, A. Optical devices based on liquid crystal photonic bandgap fibres. Opt. Express 2003, 11, 2589-2596. [CrossRef]

19. Veilleux, C.; Lapierre, J.; Jacques, B. Liquid crystal clad tapered fibers. Opt. Lett. 1986, 11, 733-735. [CrossRef]

20. Veilleux, C.; Black, R.J.; Lapierre, J. Nematic liquid crystal clad tapered optical fiber with temperature sensing properties. J. Appl. Phys. 1990, 67, 6648-6653. [CrossRef]

21. Dąbrowski, R.; Garbat, K.; Urban, S.; Woliński, T.R.; Dziaduszek, J.; Ogrodnik, T.; Siarkowska, A. Low-birefringence liquid crystal mixtures for photonic liquid crystal fibres application. Liq. Cryst. 2017, 44, 1911-1928. [CrossRef]

22. Zhang, L.; Lou, J.; Tong, L. Micro/Nanofiber Optical Sensors. Photonic Sens. 2011, 1, 31-42. [CrossRef]

23. Polynkin, P.; Polynkin, A.; Peyghambarian, N.; Mansuripur, M. Evanescent field-based optical fiber sensing device for measuring the refractive index of liquids in microfluidic channels. Opt. Lett. 2005, 30, 1273-1275. [CrossRef] [PubMed]

24. Choudhury, P.K.; Soon, W.K. On the transmission by liquid crystal tapered optical fibers. Optik 2011, 122, 1061-1068. [CrossRef]

25. Laudyn, U.; Rutkowska, K.; Rutkowski, R.T.; Karpierz, M.A.; Woliński, T.R.; Wójcik, J. Nonlinear effects in photonic crystal fibers filled with nematic liquid crystals. Cent. Eur. J. Phys. 2008, 6, 612-618. [CrossRef]

26. Jadżyn, J.; Hellemans, L.; Czechowski, G.; Legrand, C.; Douali, R. Dielectric and viscous properties of 6CHBT in the isotropic and nematic phases. Liq. Cryst. 2010, 27, 613-619. [CrossRef]

27. Siarkowska, A.; Chychłowski, M.; Budaszewski, D.; Jankiewicz, B.; Bartosewicz, B.; Woliński, T.R. Thermoand electro-optical properties of photonic liquid crystal fibers doped with gold nanoparticles. Beilstein J. Nanotechnol. 2017, 8, 2790-2801. [CrossRef] [PubMed] 
28. Budaszewski, D.; Chychłowski, M.; Budaszewska, A.; Bartosewicz, B.; Jankiewicz, B.; Woliński, T.R. Enhanced efficiency of electric field tunability in photonic liquid crystal fibers doped with gold nanoparticles. Opt. Express 2019, 27, 14260-14269. [CrossRef] [PubMed]

29. Brust, M.; Walker, M.; Bethell, D.; Schiffrin, D.J.; Whyman, R. Synthesis of thiol-derivatised gold nanoparticles in a two-phase liquid-liquid system. J. Chem. Soc. Chem. Commun. 1994, 801-802. [CrossRef]

30. Stasiewicz, K.A.; Jaroszewicz, L.R. Automatic set-up for advanced optical fiber elements manufacturing. Proc. SPIE 2005, 5952. [CrossRef]

31. Weglowski, R.; Piecek, W.; Kozanecka-Szmigiel, A.; Konieczkowska, J.; Schab-Balcerzak, E. Poly(esterimide) bearing azobenzene units as photoaligning layer for liquid crystals. Opt. Mater. 2015, 49, 224-229. [CrossRef]

32. Orlandi, S.; Benini, E.; Miglioli, I.; Evans, D.R.; Reshetnyak, V.; Zannoni, C. Doping liquid crystals with nanoparticles. A computer simulation of effects of nanoparticle shape. Phys. Chem. 2016, 18, 2428-2441. [CrossRef]

33. Urbanski, M. On the impact of nanoparticle doping on the electro-optic response of nematic hosts. Liq. Cryst. Today 2015, 24, 102-115. [CrossRef]

(C) 2019 by the authors. Licensee MDPI, Basel, Switzerland. This article is an open access article distributed under the terms and conditions of the Creative Commons Attribution (CC BY) license (http://creativecommons.org/licenses/by/4.0/). 